\title{
Un anno di letteratura nefrologica sulla nefrolitiasi
}

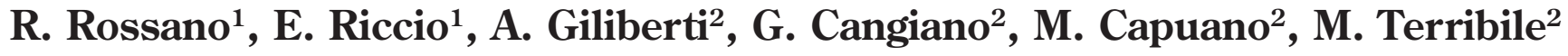 \\ ${ }^{1}$ Istituto di Nefrologia Facoltà di Medicina e Chirurgia, Università "Federico II", Napoli \\ ${ }^{2}$ U.O.C. di Nefrologia e Dialisi e Centro Calcolosi Renale, Ospedale dei Pellegrini, Napoli
}

\section{Introduzione}

La calcolosi renale è associata ad alterazioni metaboliche e fisiologiche che modificano la capacità del rene di mantenere in soluzione nelle urine sostanze che, aggregandosi, possono formare nuclei litiasici. Alla base della formazione dei calcoli delle vie urinarie vi è una serie di fattori di rischio, ampiamente studiati:

- grado di saturazione delle urine, dipendente dalla quantità di cristalli disciolti nelle urine. Uno stato di sovrassaturazione si può verificare nell'iperparatiroidismo primitivo, nell'ipercalciuria idiopatica, nell'iperossaluria, nell'acidosi tubulare renale;

- $\mathrm{pH}$ urinario, in quanto alcuni tipi di calcoli precipitano a pH critici, acido per i calcoli di cistina e di acido urico, ed alcalino per quelli di fosfato di calcio e di struvite;

- presenza di promotori della cristallizzazione;

- riduzione di inibitori della cristallizzazione, come il pirofosfato inorganico, il magnesio, il citrato, i peptidi contenenti acido glutammico ed aspartico.

$\grave{\mathrm{E}}$ in genere ampiamente condivisa l'opinione che un ruolo predominante sia svolto da ossalato, calcio ed acido urico tra le sostanze favorenti la litogenesi e dai citrati tra le sostanze inibenti la litogenesi (1).

L'ossalato presente nell'organismo deriva da due origini diverse: quella assorbita a livello intestinale, e quella generata dall'organismo stesso a partire dal metabolismo di acido ascorbico e di alcuni aminoacidi (2). In genere, molto meno del $20 \%$ dell'ossalato introdotto con la dieta è assorbito (3). Infatti una quota significativa dell'ossalato intestinale - si stima fino a 70-100 mg/giorno - viene metabolizzata e degradata da batteri normalmente presenti nel lume intestinale, come l'Oxalobacter formigens (4). Inoltre l'ossalato è in genere presente nel lume intestinale in forma legata a ioni calcio come sale insolubile ed inassorbibile. L'ossalato presente nell'organismo è un prodotto terminale, non ulteriormente metabolizzabile (2). L'unica modalità di eliminazione è quindi rappresentata dal rene, che utilizza un meccanismo di secrezione tubulare presente nel tubulo contorto prossimale. In linea teorica quindi, l'iperescrezione di ossalato con le urine (iperossaluria) può riflettere un eccesso di ossalato proveniente dall'intestino (iperassorbimento o elevati introiti dietetici) o un eccesso di ossalato generato dal metabolismo endogeno. Una volta escreto nelle urine, l'ossalato è scarsamente solubile come sale di calcio e, quando in eccesso, precipita e determina la formazione di calcoli $(1,3)$.

Il calcio introdotto con gli alimenti è in genere compreso tra i 400 ed i $1500 \mathrm{mg} /$ giorno, con una larga variabilità tra una persona e l'altra e, per una stessa persona, da un giorno all'altro $(5,6)$. Il calcio introdotto con la dieta è assorbito sia dal tenue che dal colon, principalmente nei segmenti iniziali piuttosto che in quelli distali per entrambi i tratti dell'intestino. Fattori chiave nell'assorbimento intestinale sono la quantità disponibile di vitamina $\mathrm{D}$ in forma attiva, e il tipo di alimento che veicola il calcio. In media, si stima che l'assorbimento netto di calcio, calcolato cioè sottraendo all'assorbimento assoluto la quota di calcio endogeno secreto nel lume intestinale, sia pari al $15-30 \%$ circa del calcio presente in una dieta simile a quella media normale, cioè per un introito di circa 1000 $\mathrm{mg}$ /giorno di calcio (6). In altre parole, l'escrezione urinaria di calcio rende normalmente conto del bilancio tra quota di calcio assorbita a livello intestinale e quota di calcio depositata o rilasciata dal rimaneggiamento continuo del tessuto osseo. Il preciso accoppiamento tra questi fenomeni è regolato normalmente dal paratormone, attraverso una stimolazione del riassorbimento di calcio nel tubulo distale. In linea teorica quindi, un'eccessiva escrezione urinaria di calcio (ipercalciuria) può essere sostenuta da un eccessivo assorbimento intestinale, da 


\section{Fattori di rischio non tradizionali}

\section{Sindrome metabolica}

Uno studio condotto da West et al, e pubblicato nel maggio 2008, ha evidenziato un aumento della frequenza di calcolosi renale nei pazienti con sindrome metabolica (9, 10). La American Heart Association ha stabilito che si può porre diagnosi di sindrome metabolica in presenza di almeno 3 dei seguenti 5 segni: obesità addominale (circonferenza addominale $>102 \mathrm{~cm}$ nell'uomo e di $88 \mathrm{~cm}$ nella donna), aumentata concentrazione sierica di trigliceridi ( $>150 \mathrm{mg} / \mathrm{dL})$, ridotti livelli sierici di HDL $(<40 \mathrm{mg} / \mathrm{dL}$ nell'uomo e $50 \mathrm{mg} / \mathrm{dL}$ nella donna), ipertensione arteriosa (pressione sistolica $>130 \mathrm{mmHg}$ e pressione diastolica $>85 \mathrm{mmHg}$, o utilizzo di farmaci antipertensivi), e ridotta tolleranza glucidica o diabete mellito (glicemia a digiuno $>100 \mathrm{mg} / \mathrm{dL}$ o uso di ipoglicemizzanti). In particolare, si è osservata un'associazione tra obesità e rischio di calcolosi (11-13). L'obesità determina, infatti, un aumento dell'escrezione urinaria di ossalati e di calcio, una riduzione del $\mathrm{pH}$ urinario, e una ridotta escrezione urinaria di citrati. Anche l'ipertensione arteriosa, il diabete, l'insulino-resistenza (11), e l'iperlipidemia contribuiscono alla formazione dei calcoli delle vie urinarie. Si è osservata, in pazienti dislipidemici sottoposti a terapia con atorvastatina, una riduzione dei livelli plasmatici di acido urico, attribuibile all'aumentata escrezione urinaria di quest'ultimo. Nei pazienti diabetici, si è osservata un'aumentata predisposizione alla formazione di calcoli di acido urico. Questa condizione è favorita dal basso $\mathrm{pH}$ urinario di tali soggetti, associato all'insulino-resistenza. Si può quindi affermare che il rischio di nefrolitiasi risulta direttamente proporzionale al numero di segni associati alla sindrome metabolica $(9,10)$ come si evidenzia nella Figura 1.

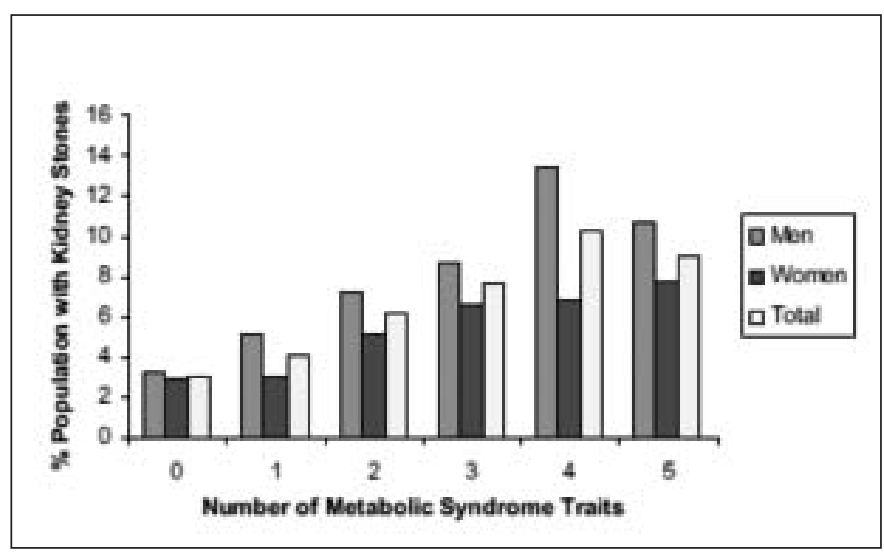

Fig. 1 - Prevalenza di nefrolitiasi in base ai segni di sindrome metabolica ed al sesso. 


\section{By-pass gastrico (RYGB)}

Sinha et al hanno dimostrato, nel 2007, la correlazione esistente tra calcolosi renale e intervento di by-pass gastrico (14-19), conseguente all'iperossaluria da aumentato assorbimento intestinale di ossalati. Infatti sono stati analizzati pazienti sottoposti a intervento di chirurgia bariatrica Roux-en-Y (RYGB) e affetti da insufficienza renale acuta (talvolta sovrapposta ad insufficienza renale cronica). La biopsia renale dimostrava, in questi pazienti, abbondanti depositi tubulari di ossalato di calcio, come da nefropatia ossalica. Alla base dell'iperossaluria enterica (14-19) vi è l'aumentato assorbimento intestinale di ossalati, che normalmente vengono assorbiti solo in minima parte grazie al loro legame con il calcio. Tuttavia, nei pazienti sottoposti a tale intervento, assistiamo ad un ridotto assorbimento degli acidi grassi, che, chelando il calcio a livello intestinale, ne impediscono il legame con gli ossalati (20).

\section{Morbo celiaco ed altre patologie intestinali}

In uno studio condotto presso l'Università degli Studi di Napoli "Federico II" da Ciacci et al (21) si è dimostrata un'associazione tra celiachia e rischio di nefrolitiasi, confermata dall'evidenza che, mentre nei pazienti celiaci in dieta libera il rischio di nefrolitiasi è maggiore rispetto alla popolazione generale, in quelli in dieta priva di glutine tale rischio risulta notevolmente ridotto. La formazione di calcoli di ossalato in corso di malattia intestinale è spiegata da un modello fisiopatologico generalmente indicato come iperossaluria intestinale, termine che definisce un aumento patologico dell'ossaluria secondario ad iperassorbimento intestinale di ossalato a livello del colon (20). In favore di tale interpretazione c'è l'osservazione che l'iperossaluria intestinale colpisce anche $\mathrm{i}$ pazienti con resezione ileale (21). Numerose evidenze dimostrano che l'iperossaluria intestinale è sostenuta da vari tipi di meccanismi tra loro concorrenti. Un primo meccanismo vede come elemento determinante il quadro malassorbitivo che causa un aumento nel lume intestinale di grassi non assorbiti. L'aumento dei grassi, a sua volta, determina una secondaria riduzione nel lume intestinale della quota di ioni calcio liberi, poiché i molti grassi intraluminali non assorbiti legano gran parte degli ioni calcio disponibili. La ridotta disponibilità di ioni calcio liberi, infine, riduce la formazione di sali di ossalato di calcio aumentando quindi la quantità di ossalato libero e disponibile per l'assorbimento (20). Un secondo meccanismo, slegato dalla interazione con grassi/calcio intraluminale, è l'aumento diretto dell'assorbimento intestinale di ossalato secondario ad eccessiva permea- bilità della parete intestinale infiammata (21). Un terzo meccanismo vede coinvolto il dismicrobismo della flora batterica dei pazienti con malattia infiammatoria intestinale che comporta la riduzione di batteri metabolizzanti l'ossalato quali l'Oxalobacter formi+genes. Infine, un meccanismo non intestinale potrebbe contribuire ad elevare l'ossaluria in corso di trattamento. Sarebbe questo il caso dei pazienti in prolungata nutrizione parenterale, la quale forse determina anche un aumento della produzione endogena di ossalati dal momento che tale nutrizione può associarsi ad iperossaluria in pazienti colectomizzati e/o con introiti dietetici ridotti o assenti. Indipendentemente dal meccanismo sottostante, l'aumento della quantità di ossalato presente nell'organismo comporta necessariamente un aumento dell'escrezione urinaria di ossalato e quindi un elevato rischio di sovrasaturazione, specie se coesistono altri fattori favorenti quali ridotti volumi urinari e/o ridotta escrezione urinaria di inibitori della cristallizzazione urinaria $(1,3)$. I calcoli di acido urico sembrano privilegiare i casi clinici con diarrea abbondante o stomie del tenue (4). In genere i calcoli di acido urico sono il risultato delle abbondanti perdite intestinali di fluidi e bicarbonati che comportano necessariamente la tendenza ad urine iperconcentrate ed acide che favoriscono la precipitazione dell'acido urico.

\section{Orlistat (Xenical)}

In uno studio condotto nel 2008 presso il Memorial Hospital di Istanbul si è dimostrato un aumento dell'assorbimento di ossalati assunti con la dieta in pazienti obesi che facevano uso di inibitori della lipasi gastro-intestinale (Orlistat) (22). L'Orlistat è un farmaco che agisce inibendo a livello gastrointestinale la lipasi pancreatica, enzima necessario per la digestione dei trigliceridi a lunga catena. Ciò determina la mancata idrolisi dei grassi alimentari in acidi grassi assorbibili, impedendone in questo modo l'assorbimento di circa il $30 \%$. Anche in questo caso, come nelle patologie intestinali (20), si ha iperossaluria da aumentato assorbimento di ossalati. Questa potrebbe essere dovuta a due principali meccanismi: all'alterato trasporto mucosale di ossalati, ed alle modifiche nella concentrazione di ioni ossalato nel lume intestinale (21). Gli acidi grassi indigeriti ed inassorbiti, insieme agli acidi biliari, reagiscono infatti con il calcio intestinale, riducendo la quota di calcio disponibile per il legame con l'ossalato (22).

\section{Consumo di fruttosio}

In un lavoro pubblicato nel 2008 Taylor e Curhan hanno osservato che l'aumentato intake di fruttosio determina 
un incremento dell'escrezione urinaria di calcio, ossalati, acido urico ed altri fattori associati al rischio di calcolosi renale $(23,24)$. La relazione esistente tra consumo di fruttosio e nefrolitiasi è stata esaminata nei Nurses' Health Study I (NHS I), Nurses' Health Study II (NHS II) e Health Professionals Follow-up Study utilizzando dei questionari per valutare l'intake di fruttosio. Si è osservato, in tutti questi studi, che l'incidenza di calcolosi renale era significativamente più elevata nei soggetti con intake di fruttosio più elevato rispetto a quelli che ne facevano minor consumo $(23,24)$. I carboidrati differenti dal fruttosio, invece, non sono risultati associati al rischio di calcolosi in nessuno studio. Questo studio suggerisce, pertanto, che il consumo di fruttosio è un fattore di rischio indipendente di nefrolitiasi.

\section{Cinacalcet}

In uno studio francese pubblicato nell'ottobre del 2007 si è valutata l'associazione tra l'utilizzo del Cinacalcet e l'aumentato rischio di nefrolitiasi (25).

Tale studio ha interessato pazienti che avevano ricevuto un trapianto renale, ai quali sono state somministrate dosi progressivamente maggiori di Cinacalcet, nel tentativo di ridurre il persistente iperparatiroidismo.

$\mathrm{Si}$ è osservato, in questi pazienti, un progressivo aumento dei livelli di calcio urinario concomitante allincremento del dosaggio del farmaco.

Il Cinacalcet potrebbe quindi essere responsabile del progressivo aumento dell'escrezione urinaria di calcio, da un lato per un ridotto riassorbimento tubulare del calcio indotto dalla riduzione dei livelli di paratormone, dall'altro per un'azione diretta sui recettori del calcio localizzati a livello del segmento spesso del tratto ascendente dell'ansa di Henle, favorendo quindi la formazione di calcoli.

\section{Idrossiapatite}

Un ulteriore fattore di rischio per lo sviluppo della calcolosi renale è stato valutato in uno studio condotto presso la Florida University, Gainesville, pubblicato nel luglio del 2008. Tale studio valutava l'associazione tra la calcolosi e la deposizione di idrossiapatite (26) sotto forma di placche subepiteliali o depositi tubulari a livello delle papille renali. Si è osservato infatti che le cellule venute a contatto con i cristalli di idrossiapatite andavano incontro a fenomeni di stress ossidativo e perossidazione lipidica (26). Questi fenomeni determinavano una up-regulation dei geni che codificano per i mediatori dell'infiammazione, che potrebbero essere responsabili della flogosi renale che si osserva nei pazienti con nefrolitiasi.

\section{Topiramato}

Uno studio condotto presso l'Università del Kentucky nel 2008 ha dimostrato che il Topiramato, un antiepilettico utilizzato anche per il trattamento dell'emicrania, è associato al rischio di nefrolitiasi (27). Infatti, questo farmaco induce una marcata riduzione dei livelli urinari di citrato, paragonabili a quelli riscontrati nell'acidosi tubulare distale. Inoltre i livelli di citrato si riducono rapidamente dopo l'inizio della terapia con Topiramato e continuano a ridursi ulteriormente nel corso della terapia.

\section{Strategie terapeutiche}

\section{Succo di limone}

Il citrato di potassio può essere utilizzato in pazienti che presentano calcoli di ossalato di calcio per aumentare il $\mathrm{pH}$ urinario e l'escrezione di citrato, fattori che riducono la formazione di cristalli di ossalato di calcio $(28,29)$. Uno studio condotto nel 2008 in C, presso la Chulalong Korn University di Bangkok, aveva come obiettivo la valutazione dell'efficacia del consumo di limonata rispetto al citrato di potassio sulla riduzione dei fattori di rischio metabolici, sullo stress ossidativo e sul danno tubulare renale nei pazienti con nefrolitiasi. Si è osservato che, a parità di effetto sulla alcalinizzazione delle urine e sulla escrezione urinaria di citrati (28-31), il succo di limone si è rivelato più efficace nel ridurre la formazione di calcoli nei pazienti predisposti. Infatti nei pazienti sottoposti a tale trattamento si è potuto osservare un incremento plasmatico di potassio, un aumento dei livelli eritrocitari di glutatione con un maggior effetto antiossidante, tale da ridurre il danno tubulare renale (28-31).

\section{Oxalate-Degrading Enzime (Oxazyme)}

In uno studio condotto a Cambridge su topi knockout, presso l'Altus Pharmaceuticals, si è valutata l'efficacia di una nuova terapia orale con una formulazione cristallina di ossalato-decarbossilasi per la riduzione delliperossaluria e del danno renale (32). Infatti, nei topi a cui veniva somministrata tale formulazione si è ottenuta la riduzione dell'escrezione urinaria dell'ossalato, principale responsabile della formazione dei calcoli. Questo enzima agirebbe a livello gastrico, ad un $\mathrm{pH}$ ottimale di 3-5, degradando l'ossalato di origine alimentare.

La Figura 2 mostra l'efficacia dell'Oxazyme nella degradazione dell'ossalato in un range di concentrazione di $0.05-2.0 \mathrm{mmol} / \mathrm{L}$ (corrispondenti a 5-200mg of ossalato della dieta).

Il trattamento, quindi, con Ox-De potrebbe rappresenta- 


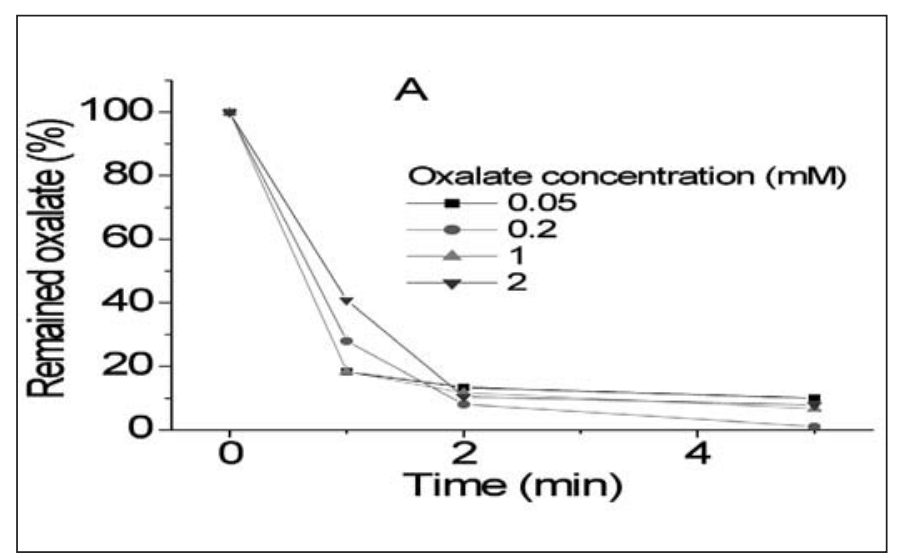

Fig. 2 - Oxazyme e degradazione dell'ossalato.

re una realistica opzione terapeutica per l'iperossaluria e per la prevenzione della nefrocalcinosi e dell'urolitiasi indipendentemente dalle cause (32).

Le Figure 3 e 4 mostrano la differente escrezione renale di ossalato in 3 gruppi di ratti: nel gruppo controllo, nel gruppo sottoposto ad una dieta ad elevato intake di ossalato (HOD), ed in quello sottoposto a dieta ad alto contenuto di ossalato ed a trattamento con Oxazyme (HOD + Oxazyme).

\section{Sevelamer}

Lieske et al, in uno studio pubblicato ad Aprile 2008, hanno valutato l'utilizzo del Sevelamer nella riduzione dei livelli urinari di ossalato e della sovrassaturazione di ossalato di calcio in pazienti con iperossaluria da cause intestinali (33). Questo agirebbe chelando gli acidi grassi ed il fosfato, e consentendo al calcio libero di legarsi all'ossalato e/o legando direttamente l'ossalato. Il Sevelamer riduce marcatamente i livelli urinari di fosforo con un effetto minore sull'ossalato urinario (33). E stato inoltre osservato un aumento dei livelli urinari di calcio ed una concomitante riduzione dei livelli urinari di citrato. Sebbene il Sevelamer non risulta essere l'agente ideale per la correzione dell'iperossaluria, le sue potenzialità nella riduzione della saturazione del fosfato di calcio devono essere oggetto di ulteriori studi.

\section{Verapamil}

Kemal et al, nel 2007, hanno dimostrato che i calcioantagonisti, oltre agli effetti già noti, prevengono la formazione di calcoli delle vie urinarie. E stato studiato l'effetto del Verapamil (34) sull'escrezione del-

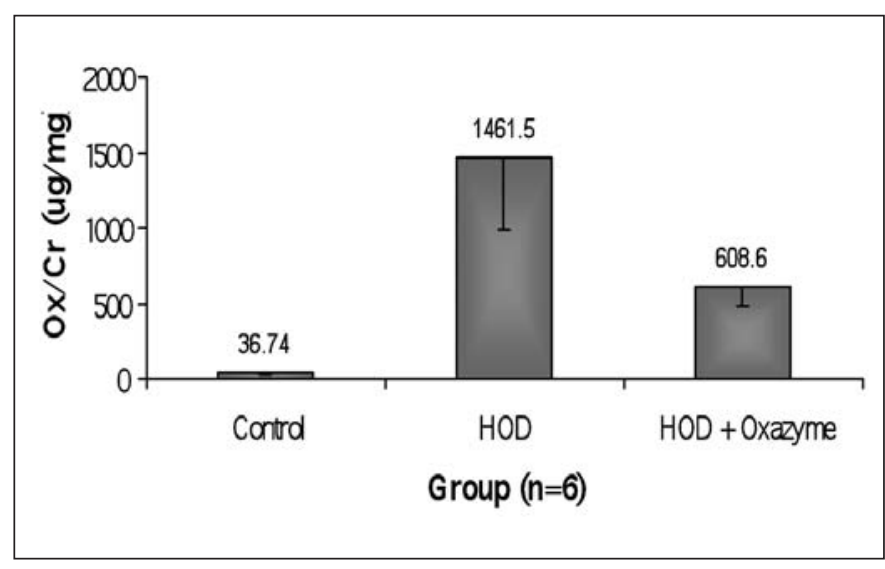

Fig. 3 - Escrezione urinaria di ossalati 6 ore dopo il pasto nei 3 gruppi di ratti studiati.

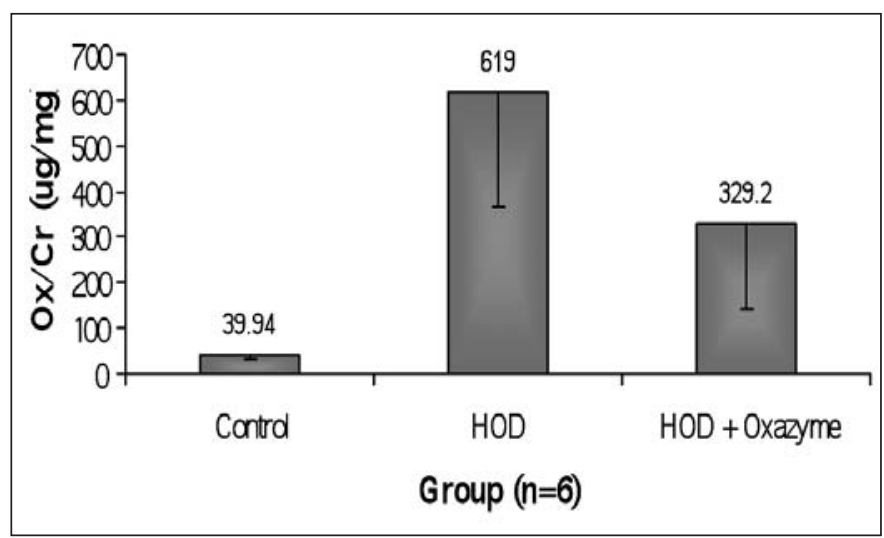

Fig. 4 - Escrezione urinaria di ossalati 12 ore dopo il pasto nei 3 gruppi di ratti studiati.

le sostanze favorenti la litogenesi, in particolare di calcio ed ossalato, e si è concluso che questo farmaco è in grado di ridurne l'escrezione urinaria. I meccanismi alla base di tale azione protettiva non sono ancora chiari, ma si suppone che questo agisca non solo a livello renale, ma anche intestinale.

\section{Rasburicase}

Il danno renale indotto dall'acido urico può essere dovuto all'ostruzione delle vie urinarie da parte di calcoli, alla precipitazione intra-tubulare di cristalli di acido urico (in caso di iper-produzione in corso di lisi tumorale), o ad una nefropatia interstiziale (come ad esempio la nefropatia iperuricemica). I calcoli di acido urico contengono un nucleo di acido urico di solito ricoperto da cristalli di ossalato di calcio, che ne determinano l'aumento delle dimensioni e che ne alterano l'aspetto radiologico (35). 


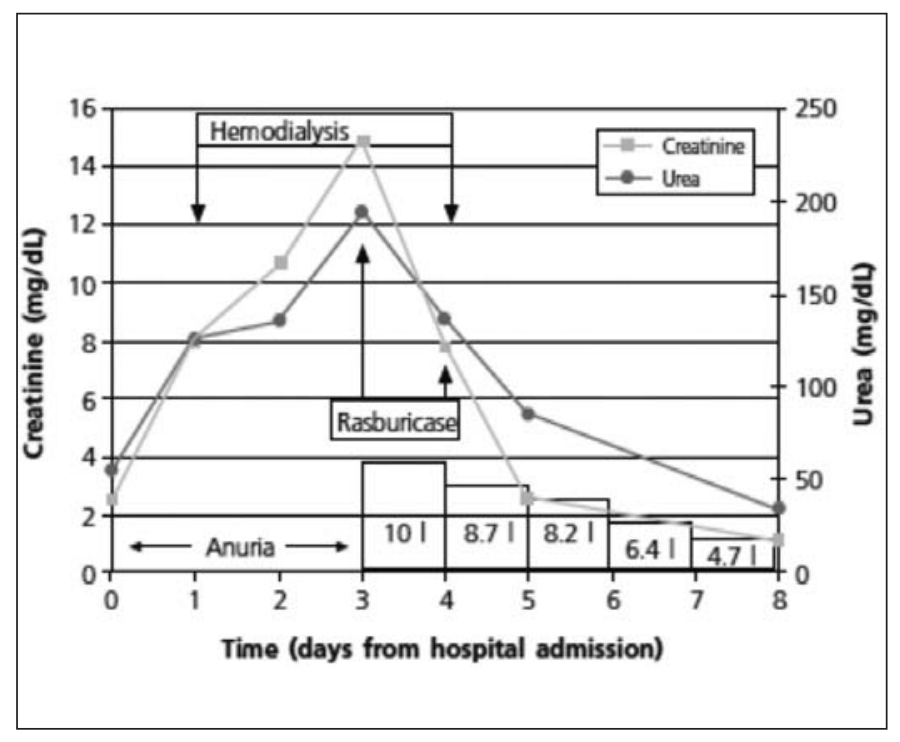

Fig. 5 - Caso 1. Funzione renale e diuresi prima e dopo terapia con Rasburicase nell'uropatia ostruttiva.

Il Rasburicase è un enzima che converte l'acido urico in allantoina, un composto più idrosolubile, e pertanto escreto più facilmente con le urine. Tale enzima viene utilizzato normalmente nella prevenzione della sindrome da lisi tumorale e nel trattamento dell'iperuricemia grave nella gotta tofacea. Segura Torre et al, in un articolo pubblicato nel 2008 (35), hanno dimostrato l'efficacia terapeutica del Rasburicase nel trattamento dell'insufficienza renale acuta e cronica associata a nefropatia ostruttiva. È stato supposto che l'enzima fosse in grado di attraversare il filtro glomerulare grazie al suo peso molecolare relativamente basso, riuscendo in tal modo a dissolvere i cristalli di acido urico ed a frammentare i calcoli nel tratto urinario, risolvendo così la nefropatia ostruttiva.

Le Figure 5 e 6 mostrano l'evoluzione della funzione renale e della diuresi dopo terapia con Rasburicase in due diversi casi.

\section{Oxalobacter formigenes}

Oxalobacter formigenes è un batterio Gram-negativo (36), anaerobio, che metabolizza l'ossalato nel tratto intestinale ed è presente nella maggior parte della popolazione normale adulta. È stato ipotizzato che la sua assenza potrebbe determinare una ridotta degradazione dell'ossalato, e quindi un suo maggior assorbimento intestinale, una maggior escrezione urinaria, responsabile dell'aumentato rischio di nefrolitiasi. Vari studi hanno infatti dimostrato che la colonizzazione con Oxalobacter formigenes è associate alla riduzione di circa il $70 \%$ del rischio di formazione ricorrente di calcoli di ossalato di calcio.

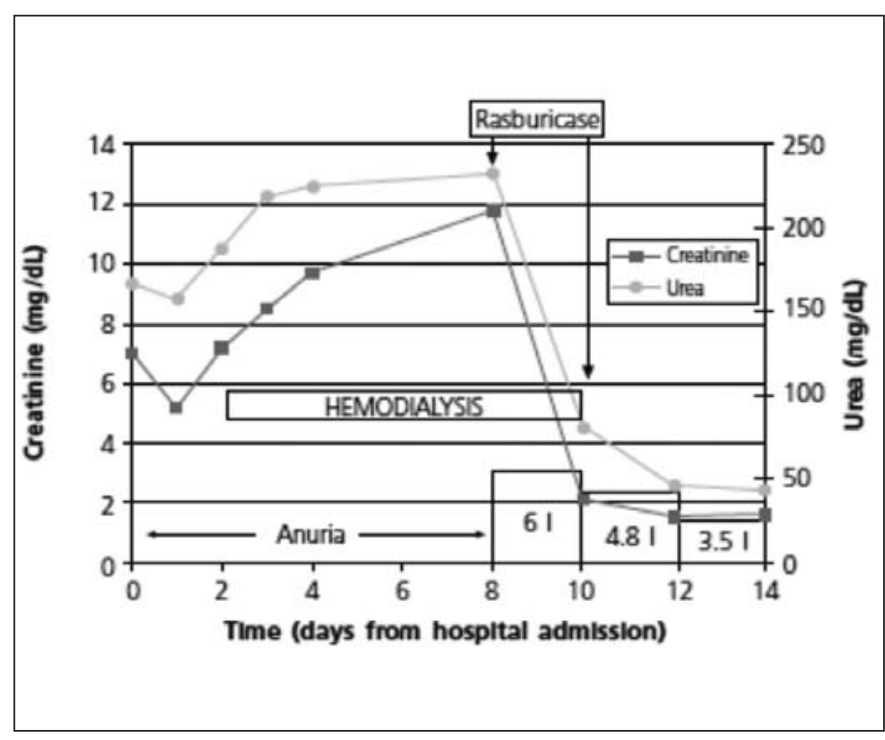

Fig. 6 - Caso 2. Funzione renale e diuresi prima e dopo terapia con Rasburicase nell'uropatia ostruttiva.

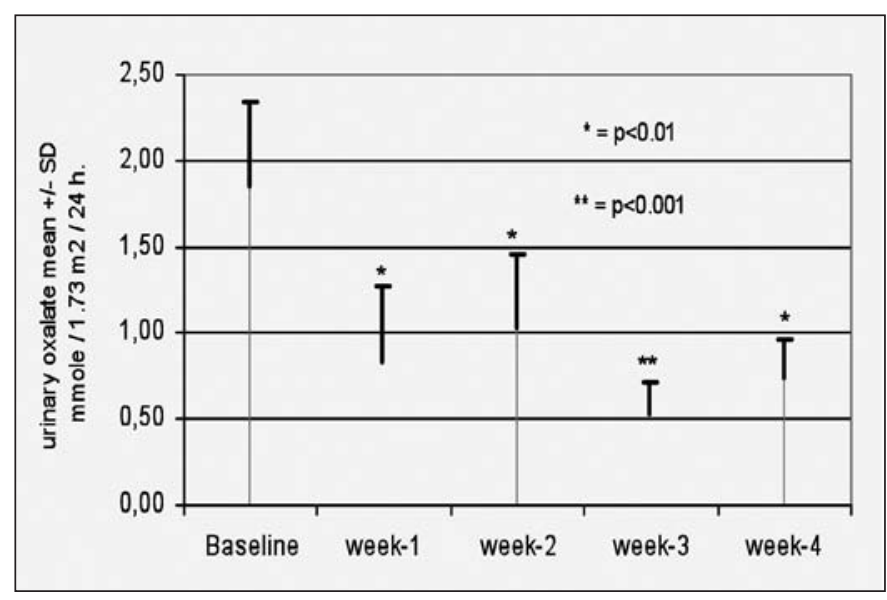

Fig. 7 - Terapia con Oxabact ed escrezione urinaria di ossalati.

\section{Oxabact}

Alcuni studi hanno valutato l'efficacia dell'Oxabact nella riduzione dell'iperossaluria primaria. Si tratta di una formulazione che agisce in modo ottimale ad un $\mathrm{pH}$ intestinale $>6,5$ aumentando la sopravvivenza dell'Oxalobacter formigenes (36), batterio della normale flora intestinale, in grado di degradare l'ossalato. In tal modo viene degradata una maggior quantità di ossalato, con conseguente riduzione dell'assorbimento intestinale e dell'escrezione urinaria.

La Figura 7 mostra la riduzione dell'escrezione urinaria di ossalati in pazienti trattati con Oxabact per 4 settimane. 


\section{Indirizzo degli Autori:}

Maurizio Terribile, MD

U.O.C. di Nefrologia e Dialisi e Centro Calcolosi Renale

Ospedale dei Pellegrini

Via Portamedina alla Pignasecca, 41

80134 Napoli, Italy

maurizio.terribile@fastwebnet.it

\section{Bibliografia}

1. Coe FL, Evan A, Worcester E. Kidney stone disease. J Clin Invest 2005; 115: 2598-608.

2. Williams AW, Wilson DM. Dietary intake, absorption, metabolism, and excretion of oxalate. Semin Nephrol 1990; 10: 2-8.

3. Holmes RP, Goodman HO, Assimos DG. Contribution of dietary oxalate to urinary oxalate excretion. Kidney Int 2001; 59: 270-6.

4. Allison MJ, Cook HM, Milne DB, Gallagher S, Clayman RV. Oxalate degradation by gastrointestinal bacteria from humans. J Nutr 1986; 116: 455-60.

5. Massry SG, Glassock RJ, ed. Massry \& Glassock's Textbook of Nephrology (4th edition), Philadelphia: Lippincott Williams \& Wilkins, 2001; 308-40.

6. Silverberg SJ. The distribution and balance of calcium, magnesium and phosphorus. Primer on the Metabolic Bone Diseases and Disorders of Mineral Metabolism, Kelseyville (CA): The American Society for Bone and Mineral Research, 1990; 30-2.

7. Yamamoto T, Moriwaki Y, Takahashi S. Effect of ethanol on metabolism of purine bases (hyoxanthine, xanthine, and uric acid). Clin Chim Acta 2005; 356: 35-57.

8. Maesaka JK, Fishbane S. Regulation of renal urate excretion: a critical review. Am J Kidney Dis 1999; 32: 917-33.

9. West B, Luke A, Durazo-Arvizu RA, Cao G, Shoham D, Kramer H. Metabolic syndrome and self-reported history of kidney stones: the National Health and $\mathrm{Nu}-$ trition Examination Survey (NHANES III) 1988-1994. Am J Kidney Dis 2008; 51(5): 741-7.

10. Sakhaee K, Maalouf NM, Metabolic syndrome and uric Acid nephrolithiasis. Semin Nephrol 2008; 28(2): 174-80.

11. Daudon M, Jungers P. Diabetes and nephrolithiasis. Curr Diab Rep 2007; 7(6): 443-8.

12. Lee SC, Kim YJ, Kim TH, Yun SJ, Lee NK, Kim WJ. Impact of obesity in patients with urolithiasis and its prognostic usefulness in stone recurrence. J Urol 2008; 179(2): 403-4.
13. Duffey BG, Pedro RN, Kriedberg C, et al. Lithogenic risk factors in the morbidly obese population. J Urol 2008; 179(4): 1401-6.

14. Duffey BG, Pedro RN, Makhlouf A, et al. Roux-en-Y gastric bypass is associated with early increased risk factors for development of calcium oxalate nephrolithiasis. J Am Coll Surg 2008; 206(3):1145-53.

15. Lieske JC, Kumar R, Collazo-Clavell ML. Nephrolithiasis After Bariatric Surgery for Obesity. Semin Nephrol 2008; 28(2): 163-73.

16. Asplin JR, Coe FL. Hyperoxaluria in kidney stone formers treated with modern bariatric surgery. J Urol 2007; $177(2): 565-9$.

17. Sinha MK, Collazo-Clavell ML, Rule A, Milliner DS, Nelson W, Sarr MG, Kumar R, Lieske JC. Hyperoxaluric nephrolithiasis is a complication of Roux-en-Y gastric bypass surgery. Kidney Int 2007; 72(1):8-10.

18. Kleinman JG. Bariatric surgery, hyperoxaluria, and nephrolithiasis: a plea for close postoperative management of risk factors. Kidney Int 2007; 72(1):100-7.

19. Nasr SH, D'Agati VD, Said SM, Stokes MB, Largoza MV, Radhakrishnan J, Markowitz GS. Oxalate Nephropathy Complicating Roux-en-Y Gastric Bypass: An Underrecognized Cause of Irreversible Renal Failure. Clin J Am Soc Nephrol 2008 Aug 13.

20. M. Cirillo, M. Iudici, F. Marcarelli, M. Laudato, F. Zincone, La nefrolitiasi nelle patologie intestinali, Giornale italiano di nefrologia/ nno 25 N. 1, 2008: 42-48-

21. Ciacci C, Spagnuolo G, Tortora R, Bucci C, Franzese D, Zingone F, Cirillo M. Urinary stone disease in adults with celiac disease: prevalence, incidence and urinary determinants. J Urol 2008;180(3): 974-9.

22. Sarica K, Akarsu E, Erturhan S, Yagci F, Aktaran S, Altay B. Evaluation of urinary oxalate levels in patients receiving gastrointestinal lipase inhibitor. Obesity (Silver Spring) 2008; 16(7): 1579-84.

23. Asselman M, Verkoelen CF. Fructose intake as a risk factor for kidney stone disease. Kidney Int 2008; 73(2): 207-12.

24. Taylor EN, Curhan GC. Fructose consumption and the risk of kidney stones. Kidney Int. 2008; 73(2): 139-40.

25. Esposito L, Rostaing L, Gennero I, Mehrenberger M, Durand D, Kamar N. Hypercalciuria induced by a high dose of cinacalcet in a renal-transplant recipient. J Urol 2007; 177 (4): 1358-62

26. Escobar C, Byer KJ, Khaskheli H, Khan SR. Apatite induced renal epithelial injury: insight into the pathogenesis of kidney stones. J Urol 2008; 180(1): 379-87.

27. Warner BW, LaGrange CA, Tucker T, Bensalem-Owen $\mathrm{M}$, Pais VM Jr. Induction of progressive profound hypocitraturia with increasing doses of topiramate. 
Urology 2008; 72(1): 29-32.

28. Penniston KL, Steele TH, Nakada SY. Lemonade therapy increases urinary citrate and urine volumes in patients with recurrent calcium oxalate stone formation. Urology 2007; 70(5): 856-60.

29. Haleblian GE, Leitao VA, Pierre SA, Robinson MR, Albala DM, Ribeiro AA, Preminger GM. Assessment of citrate concentrations in citrus fruit-based juices and beverages: implications for management of hypocitraturic nephrolithiasis. J Endourol 2008; 22(6): 1359-66.

30. Kang DE, Sur RL, Haleblian GE, Fitzsimons NJ, Borawski KM, Preminger GM, Long-term lemonade based dietary manipulation in patients with hypocitraturic nephrolithiasis. J Urol 2007; 177(4): 1358-62.

31. Tosukhowong P, Yachantha C, Sasivongsbhakdi T, Ratchanon S, Chaisawasdi S, Boonla C, Tungsanga K. Citraturic, alkalinizing and antioxidative effects of limeade-based regimen in nephrolithiasis patients. Urol Res 2008; 36(3-4): 149-55.

32. Grujic D, Salido EC, Shenoy BC, Langman CB, Mc-
Grath ME, Patel RJ, Rashid A, Mandapati S, Jung CW, Margolin AL. Hyperoxaluria Is Reduced and Nephrocalcinosis Prevented with an Oxalate-Degrading Enzyme in Mice with Hyperoxaluria. Am J Nephrol 2008; 12; 29(2): 86-93.

33. Lieske JC, Regnier C, Dillon JJ. Use of sevelamer hydrochloride as an oxalate binder. J Urol 2008; 179(4): 1407-10.

34. Kemal Sarica - Sakip Erturhan · Bulent Altay. Effect of verapamil on urinary stone-forming risk factors. Urol Res (2007) 35:23-27.

35. Segura Torre P, Borrego Utiel FJ, Pérez Del Barrio P, Gil Cunqueiro JM, Pérez Ba-asco V. Efficacy of Rasburicase therapy in obstructive nephropathy from renal calculi: a novel therapeutic. Nefrologia 2008; 28(1): 102-05.

36. Kaufman DW, Kelly JP, Curhan GC, Anderson TE, Dretler SP, Preminger GM, Cave DR. Oxalobacter formigenes may reduce the risk of calcium oxalate kidney stones. J Am Soc Nephrol 2008; 19(6): 1197-203. 